\title{
LOWER BOUNDS FOR Z-NUMBERS
}

\author{
ARTŪRAS DUBICKAS AND MICHAEL J. MOSSINGHOFF
}

\begin{abstract}
Let $p / q$ be a rational noninteger number with $p>q \geq 2$. A real number $\lambda>0$ is a $Z_{p / q}$-number if $\left\{\lambda(p / q)^{n}\right\}<1 / q$ for every nonnegative integer $n$, where $\{x\}$ denotes the fractional part of $x$. We develop several algorithms to search for $Z_{p / q}$-numbers, and use them to determine lower bounds on such numbers for several $p$ and $q$. It is shown, for instance, that if there is a $Z_{3 / 2}$-number, then it is greater than $2^{57}$. We also explore some connections between these problems and some questions regarding iterated maps on integers.
\end{abstract}

\section{An approximate multiplication problem}

Let us begin with the following problem. Starting with a positive integer $x$, we consider the map

$$
x \rightarrow\left\{\begin{array}{lll}
4 x / 3, & \text { if } x \equiv 0 & (\bmod 3) \\
(4 x+1) / 3, & \text { if } x \equiv 2 & (\bmod 3) \\
\text { STOP }, & \text { if } x \equiv 1 & (\bmod 3)
\end{array}\right.
$$

Consider the iterates of this map, starting, for instance, with $x=6$. We have $6 \rightarrow 8 \rightarrow 11 \rightarrow 15 \rightarrow 20 \rightarrow 27 \rightarrow 36 \rightarrow 48 \rightarrow 64 \rightarrow$ STOP. The reader might easily guess that the problem is the following:

- Prove that, starting with any positive integer $x$, the sequence of iterates of this map terminates.

Some simple computational experiments show that usually nothing exceptional happens with this sequence of iterates and it "behaves" properly. A simple heuristic argument suggests that a positive integer $x$ "survives" at each step with probability $2 / 3$, and so one would expect approximately $(2 / 3)^{k} x$ of the possible starting values $\leq x$ to survive at least $k$ iterations. It follows that the expected stopping time for an initial value $x$ is likely to be bounded above by $c \log x$, where $c$ is some absolute constant. For example, the sequence starting with $x=3^{k}$ terminates after $k$ iterations. Although the first author has already stated this $4 / 3$ problem at several conferences, it appears that there is currently no promising approach to its solution.

This problem may very well remind the reader of the so-called $3 x+1$ problem, where the map is $x \rightarrow x / 2$ if $x$ is even and $x \rightarrow(3 x+1) / 2$ if $x$ is odd, and the

Received by the editor January 22, 2008 and, in revised form, August 7, 2008.

2000 Mathematics Subject Classification. Primary 11K31; Secondary 11J71, 11 Y35.

Key words and phrases. $Z$-numbers, distribution mod 1.

The research of the first author was partially supported by the Lithuanian State Science and Studies Foundation. 
object is to show that for any starting integer value $x$ the iterates eventually reach the cycle $1 \rightarrow 2$. While there is considerable literature on the $3 x+1$ problem, its generalizations, and similar problems on iterated maps (see for instance [4,13,21, or the annotated surveys [14,15]), the resolution of problems like these seems to be beyond the reach of current methods. Partial results establish, for instance, lower bounds on the length of long terminating sequences [1, find lower bounds on the density of initial values $x$ for which termination is assured 11, and determine information on possible cycles in the iteration [19]. So one might suspect the $4 / 3$ problem to be quite difficult as well. The situation is similar with the so-called approximate squaring problem considered by Lagarias and Sloane [12, where the map is $x \rightarrow x\lceil x\rceil$ (throughout, $\lceil x\rceil$ designates the smallest integer $\geq x$ ), and the problem is to determine whether, starting from any rational number $x>1$, one must eventually reach an integer.

The $4 / 3$ problem is related to the distribution of the powers of $4 / 3$ modulo 1 (see the next section). More generally, given any real number $\alpha>1$ which is not an integer, we can consider the map $\mathbb{N} \rightarrow \mathbb{N}$ defined by $x \rightarrow\lceil\alpha x\rceil$, and ask the following question.

- Is it true that for any positive integer $q \geq 2$ and any $j \in\{0,1, \ldots, q-1\}$ the sequence of iterates of the map $x \rightarrow\lceil\alpha x\rceil$ contains infinitely many terms in the arithmetic progression $q k+j, k=0,1,2, \ldots$ ?

In particular, for a rational number $\alpha=p / q>1$ with $q \geq 2$, and any nonempty set $S \subset\{0,1, \ldots, q-1\}$ with $|S|<q$, we consider the map

$$
x \rightarrow \begin{cases}\lceil p x / q\rceil, & \text { if } x \equiv s \bmod q, \text { for some } s \in S, \\ \text { STOP, } & \text { otherwise. }\end{cases}
$$

Thus, when $x$ belongs to a permissible residue class mod $q$, its image under this map is near its product with $p / q$. We might describe this as another problem on approximate multiplication, and we may pose the following generalization of the 4/3 problem:

- Prove that, starting with any positive integer $x$, the sequence of iterates of the map (1.1) must terminate.

As above, it seems likely that the expected number of iterations, starting from $x$, is bounded above by $c \log _{q /|S|} x$, where $c$ is some absolute constant. The $4 / 3$ problem is then the special case with $p / q=4 / 3$ and $S=\{0,2\}$. In Proposition 3.2 of Section 3 we present a simple proof of this conjecture for sets $S$ of cardinality 1. It would be of interest to settle this general problem for at least some sets $S$ with two elements (so that $q \geq 3$ ). For example, if $p=q+1$ and $S=\{0, q-1\}$, then the map is $x \rightarrow\lceil(q+1) x / q\rceil$ if $x$ is 0 or $q-1$ modulo $q$, and STOP if $x$ belongs to one of the remaining $q-2$ residue classes.

Proving that the iteration (1.1) terminates for all $\alpha=p / q$ and all permissible sets $S$, even without effective estimates on the stopping time, would imply that for any positive real number $\lambda$, the sequence of fractional parts of $\lambda(p / q)^{n}$ with $n \geq 0$ has a limit point $w$ satisfying $w \geq \min \{|S| / p, 1 / q\}$. It would further imply that the so-called $Z$-numbers defined by Mahler, and certain generalizations, do not exist. The proof of this appears in Proposition 3.1 below. We define Mahler's $Z$-numbers in the next section, and describe their connection with these problems on iterated maps of approximate multiplication in Section 3 


\section{2. $Z$-NUMBERS}

For a real number $\lambda$, let $\lfloor\lambda\rfloor$ denote its integer part and $\{\lambda\}$ its fractional part, so $\lambda=\lfloor\lambda\rfloor+\{\lambda\}$. A number of classical problems in metric number theory deal with fractional parts of real numbers arising from certain sequences, especially sequences associated with powers of real numbers, such as $\left\{(3 / 2)^{n}\right\}$. These questions investigate the distribution of such sequences in $[0,1)$, asking, for example, if the density of the terms of such a sequence that lie in some interval $[a, b)$ of $[0,1)$ is $b-a$, for every $a$ and $b$ with $0 \leq a<b \leq 1$. Such a sequence is said to be uniformly distributed $\bmod 1$.

A well-known theorem of Koksma [10] establishes that the sequence $\left\{\alpha^{n}\right\}$ is uniformly distributed mod 1 for almost every real number $\alpha>1$, so the set of exceptions forms a set of Lebesgue measure 0. The exceptional set includes the integers of course, as well as the set of real algebraic integers $\alpha>1$ whose conjugates all lie in the closed unit disk (the sets of Pisot and Salem numbers - see [2]).

The following statements are also well-known consequences of Koksma's theorem:

- (Weyl's theorem.) For any fixed $\alpha>1$, the sequence $\left\{\lambda \alpha^{n}\right\}$ is uniformly distributed modulo 1 for almost all real $\lambda$.

- For any fixed $\lambda>0$, the sequence $\left\{\lambda \alpha^{n}\right\}$ is uniformly distributed modulo 1 for almost all real $\alpha>1$.

It is interesting to note that until fairly recently no explicit real number $\alpha$ was known for which $\left\{\alpha^{n}\right\}$ is uniformly distributed mod 1. However, in 1996 Lerma [16] showed that for any positive $\lambda$ there exists a dense set of computable numbers $\alpha>1$ such that $\left\{\lambda \alpha^{n}\right\}$ is uniformly distributed modulo 1 .

In this paper, we are interested in sequences of the form $\left\{\lambda \alpha^{n}\right\}$, with $\alpha$ a fixed, simple rational number. Here, for the case $\lambda=1$ and $\alpha=p / q$ (with $p>q \geq 2$ and $\operatorname{gcd}(p, q)=1)$, Vijayaraghavan $\left[20\right.$ showed that the sequence $\left\{(p / q)^{n}\right\}$ contains infinitely many accumulation points, although no specific accumulation point is known for any particular such $\alpha$. More generally (see [5] or [18]), this statement also holds for sequences $\left\{\lambda \alpha^{n}\right\}$, with $\lambda>0$ and $\alpha$ any real algebraic number greater than 1 , except when $\alpha$ is a Pisot number and $\lambda \in \mathbb{Q}(\alpha)$.

In 1995, Flatto, Lagarias, and Pollington [7] proved that if $p$ and $q$ are relatively prime integers with $p>q \geq 2$ and $\lambda$ is a positive real number, then

$$
\limsup _{n \rightarrow \infty}\left\{\lambda\left(\frac{p}{q}\right)^{n}\right\}-\liminf _{n \rightarrow \infty}\left\{\lambda\left(\frac{p}{q}\right)^{n}\right\} \geq \frac{1}{p} .
$$

In particular, the sequence $\left\{(3 / 2)^{n}\right\}$ has at least one accumulation point $w \geq 1 / 3$. Their work was extended further by Bugeaud in 2004 [3]. Also, an inequality similar to (2.1) for the difference of limit points of the sequence $\left\{\lambda \alpha^{n}\right\}$, where $\alpha$ is an algebraic number, was recently obtained in [6].

These bounds may be improved if one could establish that certain real numbers known as $Z$-numbers do not exist. In 1968, Mahler [17] defined a $Z$-number as a real number $\lambda>0$ with the property that $\left\{\lambda(3 / 2)^{n}\right\}<1 / 2$ for all $n \geq 0$. It follows from the consequences of Koksma's theorem cited above that the set of $Z$-numbers forms a set of measure 0 . Mahler proved that this set is in fact at most countable, and furthermore showed that the number of $Z$-numbers $z \leq x$ is $O\left(x^{\gamma}\right)$, where $\gamma=\log _{2}\left(\frac{1+\sqrt{5}}{2}\right)=0.6942 \ldots$ This estimate was improved by Flatto in 1992 [8], who used results from symbolic dynamics to show that the number of $Z$-numbers up to $x$ is $O\left(x^{\beta}\right)$, where $\beta=\log _{2}(3 / 2)=0.5849 \ldots$ 


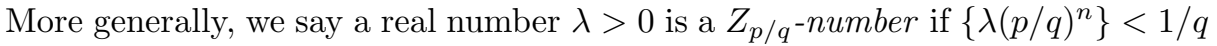
for every nonnegative integer $n$. Flatto $[8$ proved that if $\operatorname{gcd}(p, q)=1$ and $2 \leq$ $q<p<q^{2}$, then the set of $Z_{p / q}$-numbers is again at most countable, and that the number of such numbers up to $x$ is $O\left(x^{\log _{q}(p / q)}\right)$.

In this article, we develop some algorithms to search for $Z_{p / q}$-numbers for the case when $p$ and $q$ satisfy $2 \leq q<p<2 q$. It is widely believed that no such $Z_{p / q}$-numbers exist (see Proposition 3.1 below). These algorithms exploit the fact that certain congruence classes modulo powers of $q$ may be eliminated as possible values for the integer part of a $Z_{p / q}$-number. By maintaining a list of permissible residue classes for a particular $p / q$, we find that we need only check $O\left(x^{\log _{q}(p / q)+\delta}\right)$ congruence classes up to $x$, with $\delta$ a very small positive number, so the complexity of our method approaches the natural upper bound of Flatto.

Section 3 develops some properties of $Z_{p / q}$-numbers, and demonstrates how, given a $Z_{p / q}$-number $\lambda$, one may compute the sequence $\lambda(p / q)^{n}$ for $n \geq 0$ by using separate recurrence formulas for its integer and fractional parts. Section 4 describes four algorithms we developed to search for $Z_{p / q}$-numbers, and Section 5 summarizes the results of our searches. We find, for example, that no $Z_{3 / 2}$-numbers (i.e., the classical $Z$-numbers considered by Mahler) exist up to $2^{57}$, and no $Z_{4 / 3}$-numbers exist up to $10^{32}$.

\section{3. $Z_{p / q}$-NUMBERS AND ITERATED MAPS}

Suppose $\lambda$ is a $Z_{p / q}$-number, so $\left\{\lambda(p / q)^{n}\right\}<1 / q$ for each $n \geq 0$. Following Mahler [17] and Flatto [8], we first recall that the integer and fractional parts of $\lambda(p / q)^{n}$ may be computed with independent recurrence formulas if $p<q^{2}$. The following lemma describes these decoupled recurrences.

Lemma 3.1. Suppose $\lambda$ is a $Z_{p / q}$-number, with $\operatorname{gcd}(p, q)=1$ and $2 \leq q<p<$ $q^{2}$. Let $g_{n}=\left\lfloor\lambda(p / q)^{n}\right\rfloor$ and $r_{n}=\left\{\lambda(p / q)^{n}\right\}$ for $n \geq 0$, and let $e_{n}$ be the least nonnegative residue of $-p g_{n} \bmod q$. Then

$$
g_{n+1}=\frac{p g_{n}+e_{n}}{q}=\left\lceil\frac{p g_{n}}{q}\right\rceil
$$

and

$$
r_{n+1}=\frac{p r_{n}-e_{n}}{q}=\frac{p r_{n}}{q} \bmod \frac{1}{q}
$$

for $n \geq 0$.

Proof. Certainly, $0 \leq r_{n}<1 / q$ for each $n$ and $g_{n+1}+r_{n+1}=\frac{p}{q}\left(g_{n}+r_{n}\right)$. If $q \mid g_{n}$, then since $p g_{n} / q \in \mathbb{Z}$ and $p r_{n} / q<p / q^{2}<1$, we see that $g_{n+1}=p g_{n} / q$ and $r_{n+1}=p r_{n} / q$, so in fact $0 \leq r_{n}<1 / p$. If $q \nmid g_{n}$, select $e_{n}$ with $1 \leq e_{n}<q$ so that $p g_{n} \equiv-e_{n} \bmod q$. It follows that either $g_{n+1}=\left(p g_{n}-q+e_{n}\right) / q$ and $r_{n+1}=\left(p r_{n}+q-e_{n}\right) / q$, or $g_{n+1}=\left(p g_{n}+e_{n}\right) / q$ and $r_{n+1}=\left(p r_{n}-e_{n}\right) / q$. However, the first possibility implies $r_{n+1} \geq 1 / q$, so the latter case must occur, and thus in fact $e_{n} / p \leq r_{n}<\min \left\{\left(1+e_{n}\right) / p, 1 / q\right\}$. The statement follows.

The sequences $g_{n}$ and $r_{n}$ may therefore be computed by iterating the maps

$$
G(n)=\left\lceil\frac{p n}{q}\right\rceil
$$


and

$$
R(x)=\frac{p x}{q} \bmod \frac{1}{q},
$$

since $g_{n}=G^{n}\left(g_{0}\right)$ and $r_{n}=R^{n}\left(r_{0}\right)$. It is worth noting that the map $T(x)=$ $q R(x / q)=\{p x / q\}$ forms a $\beta$-transformation, a well-studied map in symbolic dynamics.

We use Lemma 3.1 to establish the connection between the approximate squaring problem of Section 1 and the question of the existence of $Z_{p / q}$-numbers.

Proposition 3.1. Let $p$ and $q$ be relatively prime positive integers, with $2 \leq q<p$, and let $S \subset\{0,1, \ldots, q-1\}$ satisfy $0<|S|<q$. If the iterated map (1.1) terminates given any positive integer $x$ as a starting value, then for every $\lambda>0$ the sequence $\left\{\lambda(p / q)^{n}\right\}$ has at least one accumulation point $w$ with $w \geq \min \{|S| / p, 1 / q\}$. Furthermore, if in addition $p<|S| q$, then no $Z_{p / q}$-number exists.

Proof. Suppose $p, q$, and $S$ satisfy the hypotheses, and suppose $\lambda$ is a positive real number for which the sequence $\left\{\lambda(p / q)^{n}\right\}$ has no accumulation point greater than or equal to $\min \{|S| / p, 1 / q\}$. By replacing $\lambda$ with $\lambda(p / q)^{m}$ with $m$ sufficiently large, we may assume that $\lambda \geq 1$ and $r_{n}:=\left\{\lambda(p / q)^{n}\right\}<\min \{|S| / p, 1 / q\}$ for all $n \geq 0$. Then $\lambda$ is a $Z_{p / q}$-number, and by Lemma 3.1 we have that $r_{n+1}=\left(p r_{n}-e_{n}\right) / q$, for some integer $e_{n} \in[0, q)$. But because (1.1) terminates with starting value $x=\lfloor\lambda\rfloor$, it follows that the sequence $\left\{e_{n}\right\}_{n \geq 0}$ contains at least $|S|+1$ different values. Thus $e_{k} \geq|S|$ for some $k \geq 0$, and so $r_{k} \geq e_{k} / p \geq|S| / p$, contradicting our assumption. The last statement follows immediately since in this case $\min \{|S| / p, 1 / q\}=1 / q$.

Next, we establish that the set $S$ cannot be a singleton.

Proposition 3.2. Let $p$ and $q$ be relatively prime positive integers, with $2 \leq q<p$, and let $S=\{s\}$ with $0 \leq s<q$. Then the iterated map (1.1) halts on every starting value $x$.

Proof. Suppose the map (1.1) does not halt on the initial input $g_{0}$, for some positive integer $g_{0}$. Then the $n$th iterate $g_{n}$ satisfies $g_{n} \equiv s \bmod q$ for each $n$, and it follows that $g_{n+1}=\left(p g_{n}+t\right) / q$, where $t$ is the least nonnegative residue of $-p s \bmod q$. Thus

$$
\begin{aligned}
g_{n} & =\left(\frac{p}{q}\right)^{n} g_{0}+\frac{t}{q}\left(1+\frac{p}{q}+\cdots+\left(\frac{p}{q}\right)^{n-1}\right) \\
& =\left(\frac{p}{q}\right)^{n}\left(g_{0}+\frac{t}{p-q}\right)-\frac{t}{p-q},
\end{aligned}
$$

and so

$$
g_{n}(p-q)+t=\left(\frac{p}{q}\right)^{n}\left(g_{0}(p-q)+t\right)
$$

for every $n \geq 0$. However, the left side is an integer for every $n \geq 0$ by hypothesis, but the right side is not for sufficiently large $n$.

We now restrict to rational numbers $p / q$ with $\operatorname{gcd}(p, q)=1$ and $2 \leq q<p<2 q$. Let $\lambda$ be a $Z_{p / q}$-number for such a rational number, and define the sequences $g_{n}$ and $r_{n}$ as in Lemma 3.1. Then from (3.2) it follows that $e_{n} \leq p r_{n}<p / q<2$, so $e_{n} \in\{0,1\}$ for each $n$. We write $\epsilon_{n}$ in place of $e_{n}$ for this case, and denote the 
binary sequence obtained from the first $n$ iterates of $G$ on $g_{0}=\lfloor\lambda\rfloor$ by $\epsilon_{0} \epsilon_{1} \cdots \epsilon_{n}$. Thus,

$$
\epsilon_{n}= \begin{cases}0 & \text { if } q \mid g_{n}\left(\text { and } 0 \leq r_{n}<\frac{1}{p}\right), \\ 1 & \text { if } q \nmid g_{n}\left(\text { and } \frac{1}{p} \leq r_{n}<\frac{1}{q}\right) .\end{cases}
$$

It follows then from (3.1) and (3.2) that

$$
g_{m}=-\frac{1}{p} \sum_{k=0}^{n-1} \epsilon_{m+k}\left(\frac{q}{p}\right)^{k}+\left(\frac{q}{p}\right)^{n} g_{m+n}
$$

and

$$
r_{m}=\frac{1}{p} \sum_{k=0}^{n-1} \epsilon_{m+k}\left(\frac{q}{p}\right)^{k}+\left(\frac{q}{p}\right)^{n} r_{m+n}
$$

for any nonnegative integers $m$ and $n$. Thus, $\{\lambda\}=r_{0}=\frac{1}{p} \sum_{k \geq 0} \epsilon_{k}\left(\frac{q}{p}\right)^{k}$, and so each interval $[n, n+1)$ with $n \in \mathbb{Z}^{+}$may contain at most one $Z_{p / q}$-number. This is in essence Mahler's proof that the number of $Z_{p / q}$-numbers is at most countable.

Note that Proposition 3.2 implies that no real number $\lambda>0$ has the property that $\left\{\lambda(p / q)^{n}\right\}<1 / p$ for all $n \geq 0$, since such a $\lambda$ would produce the constant sequence $\epsilon_{n}=0$. This fact was also observed in [7, Theorem 3.1].

One may obtain necessary conditions on the integer part $g_{0}$ of a $Z_{p / q}$-number by observing that certain sequences of bits are prohibited in the chain $\epsilon_{0} \epsilon_{1} \cdots$ obtained from such a number. Equation (3.7) implies that the bit sequence must satisfy

$$
\sum_{k=0}^{n-1} \epsilon_{m+k}\left(\frac{q}{p}\right)^{k}<\frac{p}{q}
$$

for all nonnegative integers $m$ and $n$. In particular, in Mahler's case $p / q=3 / 2$, one finds that the substring $\epsilon_{m}=\epsilon_{m+1}=1$ is disallowed, since $1+\frac{2}{3}>\frac{3}{2}$. It is straightforward to show that the number of binary sequences of length $n$ that do not contain two adjacent 1 bits is the Fibonacci number $F_{n+2}$. Each binary string $\epsilon_{0} \cdots \epsilon_{n-1}$ of length $n$ corresponds to a residue class for $g_{0}$ modulo $2^{n}$, so it follows that the number of permissible residue classes for the integer part of a $Z_{3 / 2}$-number is at most $F_{n+2}$. Since $F_{n+2} \sim \frac{1}{\sqrt{5}}\left(\frac{1+\sqrt{5}}{2}\right)^{n+2}$, one finds then that the number of $Z_{3 / 2}$-numbers up to $x$ is $O\left(x^{\log _{2}\left(\frac{1+\sqrt{5}}{2}\right)}\right)=O\left(x^{695}\right)$. This is Mahler's upper bound on the density of $Z_{3 / 2}$-numbers.

One may improve this bound by accounting for other disallowed bit strings $\epsilon_{m} \epsilon_{m+1} \cdots \epsilon_{m+n-1}$. For example, the shortest disallowed sequence of bits that does not contain 11 is 10101 , since $1+\left(\frac{2}{3}\right)^{2}+\left(\frac{2}{3}\right)^{4}=\frac{133}{81}>\frac{3}{2}$. Let $S_{n}$ denote the set of binary sequences of length $n$ which do not contain 11 or 10101 as a substring. Then if $x \in S_{n}$ and $n \geq 5$, then either $x=0 y$ with $y \in S_{n-1}$, or $x=100 y$ with $y \in S_{n-3}$, or $x=10100 y$ with $y \in S_{n-5}$. It follows that the characteristic polynomial for the recurrence governing $\left|S_{n}\right|$ is $f(x)=x^{5}-x^{4}-x^{2}-1$, and so $\left|S_{n}\right|=O\left(\beta^{n}\right)$ with $\beta=1.570147 \ldots$, the largest real root of $f(x)$. This implies that the number of $Z_{3 / 2}$-numbers up to $x$ is $O\left(x^{\log _{2} \beta}\right)=O\left(x^{.651}\right)$.

We apply this same method for the general case. It is simple to compute the residue class $\rho_{n}$ modulo $q^{n}$ for the integer part of a $Z_{p / q}$-number that corresponds to a given bit sequence $\epsilon_{0} \cdots \epsilon_{n-1}$. Certainly $\rho_{1}=0$ if $q \mid g_{0}$ and $\rho_{1} \equiv-1 / p \bmod q$ 
otherwise. In general, if $\epsilon_{1} \cdots \epsilon_{n-1}$ corresponds to the residue class $\rho_{n-1} \bmod q^{n-1}$, then from (3.1) we see that the sequence $0 \epsilon_{1} \cdots \epsilon_{n-1}$ corresponds to $\rho_{n} \equiv q \rho_{n-1} / p$ $\bmod q^{n}$ and $1 \epsilon_{1} \cdots \epsilon_{n-1}$ to $\rho_{n} \equiv\left(q \rho_{n-1}-1\right) / p \bmod q^{n}$. By computing forbidden sequences of bits and their corresponding residue classes $\bmod q^{n}$, we can drastically reduce the number of integers we need to check when searching for $Z_{p / q}$-numbers up to a given bound. We develop some algorithms in Section 4 to implement this strategy.

\section{Algorithms}

The first algorithm computes all the forbidden bit sequences for a given rational number $p / q$ up to a given length $N$, ensuring that no listed sequence occurs as a substring of another. The method employs a recursive strategy, building candidate sequences bit by bit, and at each stage considers only sequences $\epsilon_{0} \cdots \epsilon_{n-1}$ that form the prefix of a disallowed sequence by ensuring that

$$
0<\frac{p}{q}-\sum_{k=0}^{n-1} \epsilon_{k}\left(\frac{q}{p}\right)^{k}<\sum_{k \geq n}\left(\frac{q}{p}\right)^{k} .
$$

Algorithm 4.1. Compute forbidden sequences.

Input. Relatively prime integers $p$ and $q$, with $2 \leq q<p<2 q$, and a positive integer $N$.

Output. All binary sequences $\epsilon_{0} \cdots \epsilon_{n-1}$ with length $n \leq N$ with the property that $\sum_{k=0}^{n-1} \epsilon_{k}\left(\frac{q}{p}\right)^{k} \geq \frac{p}{q}$, but $\sum_{k=i}^{j} \epsilon_{k}\left(\frac{q}{p}\right)^{k-i}<\frac{p}{q}$ for any proper substring

Description. $\epsilon_{i} \cdots \epsilon_{j}$ of $\epsilon_{0} \cdots \epsilon_{n-1}$.

Step 1. Set $\epsilon_{0}=1$ and $S=\{\}$, and invoke Step 2 on $((p-q) / q, 1, q /(p-q))$.

Step 2. Given $(r, n, \delta)$, with $r=\frac{p}{q}-\sum_{k=0}^{n-1} \epsilon_{k}\left(\frac{q}{p}\right)^{k}$ and $\delta=\sum_{k \geq n}\left(\frac{q}{p}\right)^{k}=\frac{q^{n}}{p^{n-1}(p-q)}$. If $n<N$, then perform Step 3, followed by Step 4 .

Step 3. Set $\epsilon_{n}=1$. If $\epsilon_{i} \cdots \epsilon_{n} \in S$ for some $i$ with $0 \leq i<n$, then do nothing. Otherwise, test if $r \leq q^{n} / p^{n}$. If so, admit $\epsilon_{0} \cdots \epsilon_{n}$ to $S$ and output $\epsilon_{0} \cdots \epsilon_{n}$. If not, then invoke Step 2 on $\left(r-q^{n} / p^{n}, n+1, q \delta / p\right)$.

Step 4. Set $\epsilon_{n}=0$. If $r<q \delta / p$, then invoke Step 2 on $(r, n+1, q \delta / p)$.

The upper bound in (4.1) ensures that some subsequent selection of $\epsilon_{k}$ can produce a disallowed sequence. We can reduce this bound, improving the algorithm, by taking into account the maximal number of adjacent 1 bits allowed in the sequence of $\epsilon_{k}$. Let $m$ be the largest integer for which $\sum_{k=0}^{m-1}(q / p)^{k} \leq p / q$, so $m=\left\lfloor\log _{p / q}\left(\frac{q}{2 q-p}\right)\right\rfloor$. It follows that we can replace the right side of (4.1) by

$$
\sum_{k \geq n-1}\left(\frac{q}{p}\right)^{k}-\sum_{k \geq 0}\left(\frac{q}{p}\right)^{n-1+k(m+1)}=\left(\frac{q}{p}\right)^{n-1}\left(\frac{p}{p-q}-\frac{p^{m+1}}{p^{m+1}-q^{m+1}}\right) .
$$

Thus, we can replace the initial value of $\delta$ in Step 1 with this value, taking $n=1$.

Table 1 shows the 24 disallowed binary sequences produced by Algorithm 4.1 when $p / q=3 / 2$ and $N=45$. It also lists the residue class $\rho_{n} \bmod 2^{n}$ for an integer $g_{0}$ which produces this bit sequence under the iterated action of $G$. Analyzing these sequences in the same way as we estimated the size of the set $S_{n}$ of binary 
sequences that do not contain 11 or 10101, we find that the number of allowed bit sequences of length $n \geq 45$ is $O\left(\sigma^{n}\right)$, where $\sigma$ is the largest real root of $x^{45}-x^{44}-$ $x^{42}-x^{36}-x^{33}-x^{30}-x^{28}-x^{18}-x^{11}-x^{6}-1$, so $\sigma=1.500000000471 \ldots$, very near $3 / 2$. In this way, one may approach Flatto's bound of $O\left(x^{\log _{2}(3 / 2)}\right)=O\left(x^{.585}\right)$ for the number of candidate $Z_{3 / 2}$-numbers up to $x$.

TABLE 1. Forbidden sequences for $\frac{p}{q}=\frac{3}{2}$ and $N=45$, and corresponding residue classes.

\begin{tabular}{|c|c|c|}
\hline$n$ & $\epsilon_{0} \epsilon_{1} \cdots \epsilon_{n-1}$ & $\rho_{n} \bmod 2^{n}$ \\
\hline 2 & 11 & 3 \\
\hline 5 & 10101 & 25 \\
\hline 6 & 101001 & 41 \\
\hline 7 & 1010001 & 73 \\
\hline 8 & 10100001 & 137 \\
\hline 11 & 10100000101 & 1545 \\
\hline 14 & 10100000100101 & 14857 \\
\hline 23 & 10100000100100101000001 & 6871561 \\
\hline 24 & 101000001001001010000001 & 2677257 \\
\hline 25 & 1010000010010010100000001 & 11065865 \\
\hline 26 & 10100000100100101000000001 & 27843081 \\
\hline 29 & 10100000100100101000000000101 & 128506377 \\
\hline 30 & 101000001001001010000000001001 & 933812745 \\
\hline 31 & 1010000010010010100000000010001 & 1470683657 \\
\hline 32 & 10100000100100101000000000100001 & 2544425481 \\
\hline 33 & 101000001001001010000000001000001 & 396941833 \\
\hline 36 & 101000001001001010000000001000000101 & 47641582089 \\
\hline 37 & 1010000010010010100000000010000001001 & 13281843721 \\
\hline 38 & 10100000100100101000000000100000010001 & 219440273929 \\
\hline 41 & 10100000100100101000000000100000010000101 & 2006146669065 \\
\hline 42 & 101000001001001010000000001000000100001001 & 906635041289 \\
\hline 43 & 1010000010010010100000000010000001000010001 & 7503704807945 \\
\hline 44 & 10100000100100101000000000100000010000100001 & 3105658296841 \\
\hline 45 & 101000001001001010000000001000000100001000001 & 29493937363465 \\
\hline
\end{tabular}

Table 2 exhibits the forbidden sequences computed in the same way for the case $p / q=4 / 3$. Using this table, we calculate that the number of allowed bit sequences of length $n$ is $O\left(\tau^{n}\right)$, where $\tau$ is the largest real root of $x^{56}-x^{55}-x^{51}-x^{40}-x^{35}-x^{27}-$ $x^{21}-x^{17}-x^{4}-1$, so $\tau=1.333333338381 \ldots$ Likewise, Table 3 shows the disallowed sequences for $p / q=5 / 3$, and from this we find that the number of permissible sequences of length $n$ in this case is $O\left(\theta^{n}\right)$, where $\theta=1.666666705420 \ldots$ is the largest real root of $x^{33}-x^{32}-x^{31}-x^{26}-x^{24}-x^{20}-x^{18}-x^{15}-x^{13}-x^{11}-x^{6}-x^{2}-1$. Thus, the number of candidate $Z_{p / q}$-numbers again closely approximates the Flatto bound. Similar results occur for other $p / q$.

Algorithm 4.2 uses the list of forbidden bit sequences of length at most $N$ for a given rational number $p / q$ to construct the set of allowed residue classes for a $Z_{p / q^{-}}$ number $\bmod q^{N}$. Its recursive formulation allows computing these residues with very little demand on memory. In addition, encoding the disallowed bit strings in reverse order allows one to test for their presence in a candidate string with simple arithmetic operations. This is particularly fast for the case $p / q=3 / 2$, since one 
TABLE 2. Forbidden sequences for $\frac{p}{q}=\frac{4}{3}$ and $N=58$.

\begin{tabular}{ccc}
$n$ & $\epsilon_{0} \epsilon_{1} \cdots \epsilon_{n-1}$ & $\rho_{n} \bmod 3^{n}$ \\
\hline 2 & 11 & 8 \\
3 & 101 & 11 \\
4 & 1001 & 74 \\
9 & 100010001 & 9335 \\
10 & 1000100001 & 15896 \\
11 & 10001000001 & 35579 \\
12 & 100010000001 & 271775 \\
13 & 1000100000001 & 980363 \\
14 & 10001000000001 & 1511804 \\
15 & 100010000000001 & 3106127 \\
20 & 10001000000000010001 & 3236393171 \\
25 & 1000100000000001000010001 & 597152002808 \\
26 & 10001000000000010000100001 & 1726870148732 \\
27 & 100010000000000100001000001 & 32292929846 \\
28 & 1000100000000001000010000001 & 17825353728149 \\
33 & 100010000000000100001000000010001 & 1764087177790172 \\
34 & 1000100000000001000010000000100001 & 9176167933197536 \\
50 & 10001000000000010000100000001000001000100000000001 & 265707294289270648690124 \\
51 & 100010000000000100001000000010000010001000000000001 & 1940802598903593355820705 \\
56 & 10001000000000010000100000001000001000100000000000010001 & 370940368272515823983728691
\end{tabular}

TABlE 3. Forbidden sequences for $\frac{p}{q}=\frac{5}{3}$ and $N=33$.

\begin{tabular}{ccc}
$n$ & $\epsilon_{0} \epsilon_{1} \cdots \epsilon_{n-1}$ & $\rho_{n} \bmod 3^{n}$ \\
\hline 3 & 111 & 13 \\
4 & 1101 & 31 \\
5 & 11001 & 139 \\
6 & 110001 & 544 \\
8 & 11000011 & 3703 \\
10 & 1100001011 & 25573 \\
11 & 11000010101 & 163354 \\
12 & 110000101001 & 281452 \\
14 & 11000010100011 & 2584363 \\
16 & 1100001010001011 & 8961655 \\
17 & 11000010100010101 & 66357283 \\
19 & 1100001010001010011 & 669011377 \\
21 & 110000101000101001011 & 281590888 \\
23 & 11000010100010100101011 & 59536985314 \\
24 & 110000101000101001010101 & 1848277323013 \\
25 & 1100001010001010010101001 & 5237431761190 \\
26 & 11000010100010100101010001 & 35739821701138 \\
28 & 1100001010001010010101000011 & 81493406611060 \\
29 & 11000010100010100101010000101 & 1179579444449188 \\
30 & 110000101000101001010100001001 & \\
32 & 11000010100010100101010000100011 & \\
33 & 110000101000101001010100001000101 & 1797252840733135
\end{tabular}

can compute the residue of an integer modulo $2^{k}$ by simply checking the last $k$ bits of its binary representation.

Algorithm 4.2. Construct allowed residue classes.

Input. Relatively prime integers $p$ and $q$, with $2 \leq q<p<2 q$, a positive integer $N$, and a list of disallowed bit sequences for $Z_{p / q}$-numbers with length at most $N$.

Output. A sorted list of allowed residue classes modulo $q^{N}$ for the integer part of a $Z_{p / q}$-number.

Description.

Step 1. Invoke Step 2 on $(0,0,0)$.

Step 2. Given nonnegative integers $(s, r, n)$, with $r$ the residue class modulo $q^{n}$ corresponding to the sequence $\epsilon_{0} \cdots \epsilon_{n-1}$ of bits obtained by iterating $G$, and $s=\sum_{k=0}^{n-1} \epsilon_{n-1-k} q^{k}$. If $n=N$, then output $r$. Otherwise, first set $r^{\prime}$ to be the least nonnegative residue of $q r / p \bmod q^{n+1}$ and invoke Step 2 on $\left(q s, r^{\prime}, n+1\right)$. Then for each forbidden bit string $\epsilon_{0} \cdots \epsilon_{k-1}$ with 
length $k \leq n+1$, test if the last $k$ bits of $q s+1$ match $\epsilon_{k-1} \cdots \epsilon_{0}$. If none matches, then set $r^{\prime \prime}$ to be the least nonnegative residue of $(q r-1) / p$ $\bmod q^{n+1}$ and invoke Step 2 on $\left(q s+1, r^{\prime \prime}, n+1\right)$.

Step 3. Sort the output into ascending order.

The sorted list of allowed residue classes produced by Algorithm 4.2 is employed by Algorithm 4.3 to search for $Z_{p / q}$-numbers.

Algorithm 4.3. Search for $Z_{p / q}$-numbers.

Input. Relatively prime integers $p$ and $q$, with $2 \leq q<p<2 q$, positive integers $N, B, N_{1}$, and $N_{2}$, with $N \leq N_{1} \leq N_{2}$ and $B \geq q^{N}$, and a list $R_{N}$ of allowed residue classes for $Z_{p / q}$-numbers modulo $q^{N}$.

Output. Two sets of integers. $W_{1}$ contains all integers $x \leq B$ which require at least $N_{1}$ iterations of $G$ before reaching a disallowed residue class:

$$
\begin{array}{r}
W_{1}=\left\{x \leq B: G^{n}(x) \bmod q^{N} \in R_{N} \text { for } 0 \leq n<m,\right. \\
\left.G^{m}(x) \bmod q^{N} \notin R_{N}, \text { and } N_{1} \leq m \leq N_{2}\right\} .
\end{array}
$$

$W_{2}$ contains all integers $x \leq B$ which do not reach a disallowed residue class after $N_{2}$ iterations of $G$ :

$$
W_{2}=\left\{x \leq B: G^{n}(x) \bmod q^{N} \in R_{N} \text { for } 0 \leq n \leq N_{2}\right\} .
$$

Description.

Step 1. For each residue class $r \in R_{N}$, perform Step 2.

Step 2. For each positive integer $g_{0} \equiv r \bmod q^{N}$ with $x \leq B$, set $k=0$, and perform Step 3.

Step 3. If $k=N_{2}$, admit $g_{0}$ to $W_{2}$. Otherwise, increment $k$ and compute $g_{k}=$ $G\left(g_{k-1}\right)$. Let $\rho_{k}$ be the least nonnegative residue of $g_{k} \bmod q^{N}$, and test if $\rho_{k} \in R_{N}$ by using a binary search. If so, repeat Step 3; if not, and $k \geq N_{1}$, then admit $g_{0}$ to $W_{1}$.

Algorithm 4.3 becomes less efficient as $p / q$ approaches 2 , since relatively few residue classes are excluded for such values. It is much more practical in this case to check each residue class that corresponds to a binary sequence of a particular length. Algorithm 4.4 implements this strategy. Given $p / q$ and an integer $N$, it dynamically computes the $2^{N}$ residue classes $\bmod q^{N}$ that correspond to binary sequences of length $N$ by using a recursive method. It checks numbers in these congruence classes as candidates for integer parts of $Z_{p / q}$-numbers, computing the action of $G$ on each until finding a value of $e_{n} \geq 2$. With this strategy, there is no need to record the permissible residue classes in a lengthy list, and consequently the memory requirement for Algorithm 4.4 is just $O(N)$, in contrast to Algorithm 4.3. where the list $R_{N}$ requires $O\left((p / q)^{N}\right)$ space. Of course, in compensation Algorithm 4.4 has a somewhat worse time complexity, checking $O\left(x^{\log _{q} 2}\right)$ congruence classes up to $x$, whereas Algorithm 4.3 tests $O\left(x^{\log _{q}(p / q)+\delta}\right)$ for a small $\delta>0$. However, the low overhead of Algorithm 4.4 makes it much more practical when $p / q$ is near 2 .

Algorithm 4.4. Search for $Z_{p / q}$-numbers (low memory requirement).

Input. Relatively prime integers $p$ and $q$, with $3 \leq q<p<2 q$, positive integers $N, N_{1}$, and $N_{2}$, with $N \leq N_{1} \leq N_{2}$. 
Output. Two sets of integers. $W_{1}$ contains all integers $x \leq q^{N}$ for which the sequence $e_{0} e_{1} \cdots$ associated with the iterated action of $G$ has the property that $e_{n} \in\{0,1\}$ for $0 \leq k<n, e_{n} \geq 2$, and $N_{1} \leq n<N_{2}$. $W_{2}$ contains all integers $x \leq q^{N}$ which produce a binary sequence of length at least $N_{2}$.

Description.

Step 1. Compute $p^{-1} \bmod q^{n}$ for $1 \leq n \leq N$, and invoke Step 2 on $(0,0)$.

Step 2. Given $(x, n)$, where $0 \leq x<q^{n}$ and $x$ produces a binary sequence $s$ of length $n$ under the iterated action of $G$. If $n=N$, then invoke Step 3 on $x$. Otherwise, compute $x^{\prime}=q x / p \bmod q^{n+1}$ and $x^{\prime \prime}=(q x-1) / p$ $\bmod q^{n+1}$, so that $x^{\prime}$ corresponds to the bit sequence $0 s$, and $x^{\prime \prime}$ to $1 s$. Invoke Step 2 on $\left(x^{\prime}, n+1\right)$ and $\left(x^{\prime \prime}, n+1\right)$.

Step 3. If $x=0$, then set $g_{0}=q^{N}$; otherwise set $g_{0}=x$. Compute $g_{n+1}=G\left(g_{n}\right)$ for successive values of $n$ until either the integer $e_{n}$ from (3.1) satisfies $e_{n} \geq 2$, or $n=N_{2}$. In the former case, admit $g_{0}$ to $W_{1}$ if $n \geq N_{1}$. In the latter case, admit $g_{0}$ to $W_{2}$.

Note that since Algorithm 4.4 does not exclude any binary sequences, it determines all starting values $x \leq q^{N}$ for which the map (1.1), with $S=\{0, s\}$ and $s \equiv-1 / p \bmod q$, requires at least $N_{1}$ iterations.

In Section 5 we describe our implementations of these algorithms, and the results we obtain with them using a number of values for $p$ and $q$.

\section{RESUlTS}

Algorithms 4.1 and 4.2 were implemented in Mathematica, and Algorithms 4.3 and 4.4 were written in $\mathrm{C}++$, using the GMP multiprecision library [9] to handle arithmetic with large integers. We also implemented a special version of Algorthm 4.3 in $\mathrm{C}++$ for the case $p / q=3 / 2$ to take advantage of two opportunities for optimization. First, one can compute the residue of a positive integer mod $2^{k}$ very easily by selecting the final $k$ bits of the representation for the integer, and this is much faster than the division operation one must perform in the general case. Second, each of the permissible residues in $R_{N}$ can be stored using the G5 PowerPC's 64 bit long long data type, so that binary search can be performed natively. This saves on both time and space.

We ran Algorithm 4.3 for rational numbers $p / q$ with $2 \leq q \leq 6$ and $p / q<$ 1.75. In each case, we chose $N$ so that the program would require somewhat less than one gigabyte of memory, and then chose the bound $B$ to control the total CPU time required for the computation. Each computation was divided among $P$ processors, choosing $P$ so that each process could run overnight. The computation was divided by providing each processor with a subset of approximately $\left|R_{N}\right| / P$ residue classes from $R_{N}$ to check in Step 1 of Algorithm 4.3. Each search was completed on a network of dual $2.5 \mathrm{GHz}$ PowerMac computers, each with two gigabytes of memory, housed at the center for Interdisciplinary Research in the Mathematical and Computational Sciences (IRMACS) at Simon Fraser University.

Algorithm 4.4 is more efficient when $p / q$ is near 2 , and we used it for searches with the rational numbers $p / q=7 / 4,9 / 5$, and $11 / 6$. For example, for $p / q=$ $7 / 4$, Algorithm 4.4 requires about 25 CPU-days to search through $B=4^{38}$; using 
Algorithm 4.3 with $N=30$ would require 727 megabytes and about 60 CPUdays to check up to this same number. For $p / q=9 / 5$, Algorithm 4.3 with the reasonable choice $N=29$ would require about 35 times the CPU time needed by Algorithm 4.4 for testing through $5^{37}$. Each of the computations with Algorithm 4.4 was split among $P=2^{k}$ processors, with each processor testing all the bit sequences of length $N$ that terminate with a particular bit string of length $k$.

Table 4 summarizes the results from Algorithm 4.3. For each $p$ and $q$, this table lists the maximum length $N$ of the forbidden sequences we accounted for, the size $\left|R_{N}\right|$ of the list of allowed residues mod $q^{N}$, the bound $B$ on the numbers we checked, the threshold $N_{1}$ for admission to the set $W_{1}$ described in Algorithm 4.3. the maximum number $M$ of iterations of $G$ required for a positive integer $g_{0} \leq B$ before a forbidden residue is reached, the amount of memory required per process (in megabytes), and the total CPU time needed to complete the search. Each search used $N_{2}=5000$, but no number we tested required nearly this many iterations before reaching a forbidden residue class.

TABLE 4. Summary of searches for $Z_{p / q}$-numbers from Algorithm 4.3 .

\begin{tabular}{rrrccrrrc}
$p$ & $q$ & $N$ & $\left|R_{N}\right|$ & $B$ & $N_{1}$ & $M$ & Memory & CPU time \\
\hline 3 & 2 & 44 & 86824021 & $2^{57}$ & 121 & 137 & $700 \mathrm{Mb}$ & 54.3 days \\
4 & 3 & 58 & 32726950 & $10^{32}$ & 87 & 92 & $929 \mathrm{Mb}$ & 23.8 days \\
5 & 3 & 33 & 32048800 & $3^{42}$ & 72 & 80 & $911 \mathrm{Mb}$ & 42.1 days \\
5 & 4 & 73 & 21990987 & $4^{81}$ & 94 & 98 & $981 \mathrm{Mb}$ & 38.5 days \\
6 & 5 & 85 & 12401932 & $10^{64}$ & 102 & 104 & $573 \mathrm{Mb}$ & 11.7 days \\
7 & 5 & 48 & 17694354 & $10^{38}$ & 66 & 69 & $799 \mathrm{Mb}$ & 13.5 days \\
8 & 5 & 36 & 27608820 & $5^{42}$ & 57 & 59 & $791 \mathrm{Mb}$ & 13.0 days \\
7 & 6 & 98 & 8839228 & $10^{81}$ & 112 & 115 & $559 \mathrm{Mb}$ & 10.8 days
\end{tabular}

Table 5 presents our results on the searches performed by Algorithm 4.4. In this case, the set $W_{1}$ contains all integers $g_{0}$ which produce a binary sequence of length at least $N_{1}$, but many of these sequences contain forbidden substrings. For example, for $p / q=7 / 4$, our computation detects 65631 integers requiring at least 61 iterations, but only 18 of these have no disallowed substrings. Naturally, the ratio of permissible sequences improves as $p / q \rightarrow 2$ : For $p / q=11 / 6$, we find that 111 of the 9615 integers placed in the set $W_{1}$ by Algorithm 4.4 are allowable. The value of $M$ in Table 5 lists the length of the longest permissible sequence found in the search; the value of $M^{\prime}$ is the length of the longest binary sequence constructed without regard to forbidden substrings.

TABLE 5. Summary of searches for $Z_{p / q}$-numbers from Algorithm 4.4.

\begin{tabular}{cccccccc}
$p$ & $q$ & $N$ & $B$ & $N_{1}$ & $M$ & $M^{\prime}$ & CPU time \\
\hline 7 & 4 & 38 & $4^{38}$ & 61 & 63 & 74 & 24.9 days \\
9 & 5 & 37 & $5^{37}$ & 55 & 60 & 64 & 12.5 days \\
11 & 6 & 37 & $6^{37}$ & 53 & 56 & 59 & 12.7 days
\end{tabular}

The value of $B$ in each row of Tables 4 and 5 thus represents a lower bound on a $Z_{p / q}$-number, for the corresponding $p$ and $q$. Moreover, since our method saves the integers $g_{0}$ giving rise to particularly long iterations, for each $p$ and $q$ in 
Tables 4 and 5 we may construct a real number $\lambda \leq B$ for which $\left\{\lambda(p / q)^{n}\right\}<1 / q$ for $1 \leq n<M$, with $M$ as large as possible. We summarize our results in the following theorem.

Theorem 5.1. Suppose $\lambda \geq 1$ is a real number, let $p$ and $q$ be integers appearing in Table 4 or Table 5, and let $B$ and $M$ be the corresponding values appearing in the respective table. If $\lambda \leq B$, then $\left\{\lambda(p / q)^{n}\right\} \geq 1 / q$ for some $n \leq M$. Further, these bounds are best possible.

We describe some additional results of the calculations. In the case $p / q=3 / 2$, the longest permissible sequence obtained by iterating the map $G$ on an integer $g_{0} \leq 2^{57}$ has length 137 , achieved by $g_{0}=9850069454682037$. The sequence of bits produced by the recurrence (3.1) on this initial value is

\section{0 \\ 10100000000010000100000001010000000000000010000100010000010100000101.}

Note that the final eleven bits in this sequence form a forbidden sequence from Table 1. Using these bits and equation (3.7) with $m=0$ and $n=136$, we may construct the corresponding fractional part $r_{0}$, after selecting an arbitrary value for $r_{136}$ from the interval $[0,1 / 3)$. Using $r_{136}=1 / 4$, we find

$$
r_{0}=\frac{30765342612782061154898125372486081761716812027411380564722386987}{77355401014542844188348446843727534965514746256921793516785161121},
$$

SO

$$
\lambda=9850069454682037.3977142152879301251437438446278188 \ldots
$$

Figure 1 shows the sequence of fractional parts $\left\{\lambda(3 / 2)^{n}\right\}$ for $0 \leq n \leq 137$ for this number. This figure has a solid horizontal line at $y=1 / 2$ and a dashed line at $y=1 / 3$, so one sees that $\left\{\lambda(3 / 2)^{n}\right\}<1 / 2$ for $0 \leq n \leq 136$. Also, in view of (3.5), the ones in the bit sequence correspond to data points in the figure between the two horizontal lines, and the zeros correspond to data points below the dashed line.

Table 6 displays data for some other fractions. For each number $p / q$, it lists the integer part $g_{0}$ of the smallest real number $\lambda \leq B$ for which $\left\{\lambda(p / q)^{n}\right\}<1 / q$ for $n<M$, where the value of $B$ and $M$ is shown in Table 4 or 5 . In each case, a value for $\lambda$ may be computed in the same way as (5.1) by using (3.1), (3.5), and (3.7).

TABLE 6 . Optimal $g_{0} \leq B$ for each rational number $p / q$.

\begin{tabular}{ll}
$p / q$ & $g_{0}$ \\
\hline $3 / 2$ & 9850069454682037 \\
$4 / 3$ & 61847826254741196726720207977430 \\
$5 / 3$ & 9931931383861655226 \\
$5 / 4$ & 1029084555245225919093826819827674406464117748531 \\
$7 / 4$ & 25952831572039021537801 \\
$6 / 5$ & 8994619510296693312812947128690774219845060704061529581452484375 \\
$7 / 5$ & 15551816577729142052619310042360828125 \\
$8 / 5$ & 65858752963792216215705439453 \\
$9 / 5$ & 26993193177085025815813125 \\
$7 / 6$ & 277924220996503446576589519136710659529380223751941967479283363017060765374433668 \\
$11 / 6$ & 38877275884521480715752426157
\end{tabular}




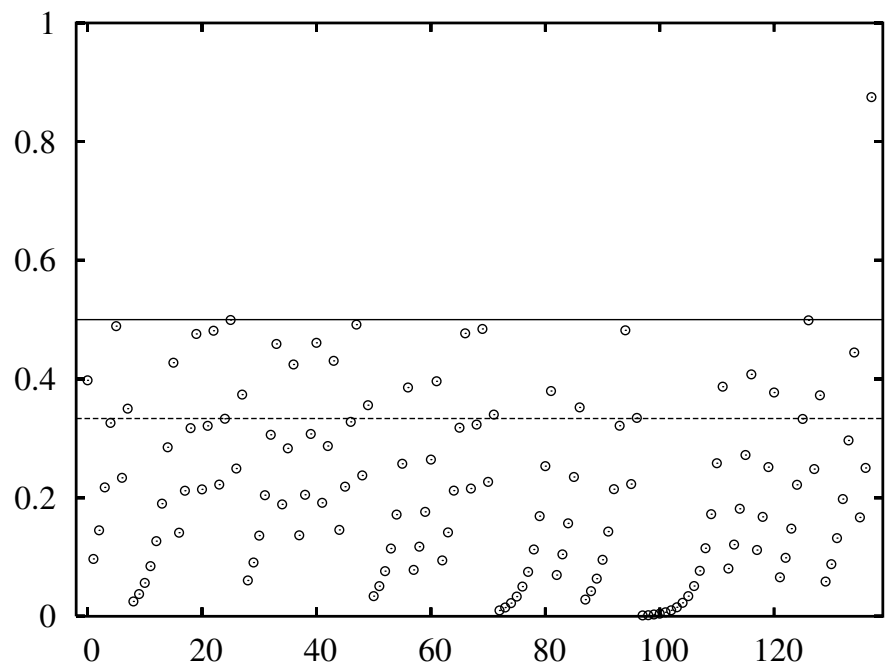

Figure 1. Fractional parts of $\lambda(3 / 2)^{n}$ for $0 \leq n \leq 137$, with $\lambda$ given by (5.1).

\section{ACKNOWLEDGMENTS}

We thank IRMACS at Simon Fraser University for computational resources, and the referee for several helpful comments and suggestions.

\section{REFERENCES}

[1] D. Applegate and J. C. Lagarias, Lower bounds for the total stopping time of $3 x+1$ iterates, Math. Comp. 72 (2003), no. 242, 1035-1049. MR1954983(2004a:11016)

[2] M.-J. Bertin, A. Decomps-Guilloux, M. Grandet-Hugot, M. Pathiaux-Delefosse, and J.-P. Schreiber, Pisot and Salem numbers, Birkhäuser, Basel, 1992. MR1187044 (93k:11095)

[3] Y. Bugeaud, Linear mod one transformations and the distribution of fractional parts $\left\{\xi(p / q)^{n}\right\}$, Acta Arith. 114 (2004), no. 4, 301-311. MR.2101819 (2005h:11163)

[4] P. Collet and J.-P. Eckmann, Iterated Maps on the Interval as Dynamical Systems, Birkhäuser, Boston, 1980. MR613981 (82j:58078)

[5] A. Dubickas, There are infinitely many limit points of the fractional parts of powers, Proc. Indian Acad. Sci. Math. Sci. 115 (2005), no. 4, 391-397. MR2184199 (2006f:11090)

[6] _ Arithmetical properties of powers of algebraic numbers, Bull. London Math. Soc. 38 (2006), no. 1, 70-80. MR2201605 (2006i:11080)

[7] L. Flatto, J. C. Lagarias, and A. D. Pollington, On the range of fractional parts $\left\{\xi(p / q)^{n}\right\}$, Acta Arith. 70 (1995), no. 2, 125-147. MR1322557 (96a:11073)

[8] L. Flatto, $Z$-numbers and $\beta$-transformations, Symbolic Dynamics and its Applications (New Haven, CT, 1991) (P. Walters, ed.), Contemp. Math., vol. 135, Amer. Math. Soc., Providence, RI, 1992, pp. 181-201. MR 1185087 (94c:11065)

[9] GMP: The GNU multiple precision arithmetic library. www.swox.com/gmp.

[10] J. F. Koksma, Ein mengentheoretischer Satz über die Gleichverteilung modulo Eins, Compositio Math. 2 (1935), 250-258. MR 1556918

[11] I. Krasikov and J. C. Lagarias, Bounds for the $3 x+1$ problem using difference inequalities, Acta Arith. 109 (2003), no. 3, 237-258. MR1980260(2004i:11020)

[12] J. C. Lagarias and N. J. A. Sloane, Approximate squaring, Experiment. Math. 13 (2004), no. 1, 113-128. MR2065571 (2005c:11098)

[13] J. C. Lagarias, The $3 x+1$ problem and its generalizations, Amer. Math. Monthly 92 (1985), no. 1, 3-23. MR777565 (86i:11043) 
[14] - The 3x+1 Problem: An Annotated Bibliography (2008). arXiv:math/0309224v11.

[15] - The 3x+1 Problem: An Annotated Bibliography, II (2008). arXiv:math/0608208v4.

[16] M. A. Lerma, Construction of a number greater than one whose powers are uniformly distributed modulo one, 1996. http://math.northwestern.edu/ mlerma/papers.

[17] K. Mahler, An unsolved problem on the powers of 3/2, J. Austral. Math. Soc. 8 (1968), 313321. MR0227109 (37:2694)

[18] Ch. Pisot, Répartition (mod 1) des puissances successives des nombres réels, Comment. Math. Helv. 19 (1946), 153-160. MR0017744 (8,194c)

[19] J. Simons and B. de Weger, Theoretical and computational bounds for $m$-cycles of the $3 n+1$ problem, Acta Arith. 117 (2005), no. 1, 51-70. MR2110503 (2005h:11049)

[20] T. Vijayaraghavan, On the fractional parts of the powers of a number, I, J. London Math. Soc. 15 (1940), 159-160. MR0002326 (2:33e)

[21] G. J. Wirsching, The dynamical system generated by the $3 n+1$ function, Lecture Notes in Math., vol. 1681, Springer-Verlag, Berlin, 1998. MR.1612686 (99g:11027)

Department of Mathematics and Informatics, Vilnius University Naugarduko 24, LT-03225 Vilnius, Lithuania

E-mail address: arturas.dubickas@mif.vu.lt

Department of Mathematics, Davidson College, Davidson, North Carolina 28035-6996

E-mail address: mimossinghoff@davidson.edu 